\title{
Enhancing Human Mobility Exoskeleton Comfort Using Admittance Controller
}

\author{
AleXANDRE RABASEDA, EMELIE SEguin, MARC DOUMIT \\ Mechanical Engineering Department at the University of Ottawa, 141 Louis-Pasteur Private \#159, \\ Ottawa, ON K1N 6N5, CANADA
}

\begin{abstract}
Human mobility exoskeletons have been in development for several years and are becoming increasingly efficient. Unfortunately, user comfort was not always a priority design criterion throughout their development. To further improve this technology, exoskeletons should operate and deliver assistance without causing discomfort to the user. For this, improvements are necessary from an ergonomic point of view. The device's control method is important when endeavoring to enhance user comfort. Exoskeleton or rehabilitation device controllers use methods of control called interaction controls (admittance and impedance controls). This paper proposes an extended version of an admittance controller to enhance user comfort. The control method used consists of adding an inner loop that is controlled by a proportional-integral-derivative (PID) controller. This allows the interaction force to be kept as close as possible to the desired force trajectory. The force-tracking admittance controller modifies the actuation force of the system in order to follow both the desired motion trajectory and the desired relative force between the user and the exoskeleton.
\end{abstract}

Keywords-Mobility assistive device, exoskeleton, force-tracking admittance controller, user comfort. Received: April 30, 2020. Revised: January 29, 2021. Accepted: March 7, 2021. Published: March 30, 2021.

\section{Introduction}

$\mathrm{E}^{\mathrm{x}}$ XOSKELETONS are mechatronic systems dedicated to enhancing human physical capacities or assisting the physical rehabilitation of a patient after an injury or surgery. In many industries, an exoskeleton can also be found as an essential tool to facilitate and improve the working conditions of laborers. Thanks to technological progress, more and more human mobility assistive devices are available and their performance has improved significantly. Until now, the main focus has been to develop a system that is functional; however, insufficient attention has been dedicated to the comfort of the user. In order to improve this technology and its acceptance among users, it is necessary to ensure that the system does not cause discomfort. Intuitively, the elderly or people undergoing rehabilitation must be assisted delicately.

The objective of this research is to enhance the comfort and ergonomics of these mobility assistive devices. This can be achieved by improving the physical interface between the user and the device, such as the development of a physical interface composed of a smart material [1]. Alternatively, the comfort and ergonomics can be enhanced by developing a controller that reduces the relative movements and forces between the device and the user.

The control methods used are called interaction control methods, which are composed of impedance and admittance controllers. However, it has been demonstrated in the literature that these controllers are not highly effective when interacting with an unpredictable and deformable environment, such as the human body. Several extensions of interaction control have been developed in order to interact with an unknown environment [2].
During this research, a force-tracking admittance controller was designed in order to estimate and control the interaction force between the system and the human limb. This method nvolved adding a PID controller to modify the trajectory of the actuator and maintain the desired interaction force. This control method was then tested with simulations on a three-dimensional (3D) model of a test bench to study user comfort. The results show that the force-tracking admittance controller that uses an internal loop driven by a PID controller reduces the relative force between the exoskeleton and the human body and thus improves user comfort.

This paper is organized as follows. First, different methods in interaction robotics used in the literature are detailed. Then, the force-tracking admittance controller model is developed, and the estimation of system parameters is presented. Finally, several simulations are made to demonstrate the efficiency of the controller.

\section{Control Methods in InteractioQ Robotics}

Different control methods exist in robotics. Depending on the robot type or the task to be achieved, the control method has to be chosen correctly. Indeed, each method achieves a certain objective. In the classic robotics industry, the most common methods are position and force controls. These methods are often adapted to control a welding or assembly robot, and are simple to implement in a known environment.

Other control methods were developed as technology progressed and as applications were diversified. Specifically, robots that interact with the human body need appropriate control methods [3], which are called interaction controls. There are several types of interaction controls: the most widely known being admittance and impedance controls.

Admittance control has been developed in response to the development of human interaction robotics. Interaction control methods have established a dynamic relation between force and position variables [4]. For admittance control, the input is force and the controller output is velocity or position. This model can 
be written in the following form:

$$
V=Y . F \quad \text { or } \quad X=Y . F
$$

where $\mathrm{F}$ is the force applied on the robot, and $\mathrm{V}, \mathrm{X}$ and $\mathrm{Y}$ are velocity, position, and the transfer function, respectively. Moreover, an admittance model commonly includes mass, spring and damper components. When the force is applied to the robot, the controller calculates the virtual corresponding position or velocity. Then, the virtual variables are used to control the real system. The virtual dynamics transfer function for admittance is given by:

$$
Y=1 /\left(m_{y} \cdot s^{2}+b_{y} . s+k_{y}\right)
$$

where $m_{y}$ represents the virtual inertia, $b_{y}$ represents the virtual damper coefficient and $k_{y}$ is the virtual stiffness. The error between the virtual output variable and real velocity or position is fed to the PID controller and is used to control the robot's joints. A simplified block diagram for an admittance controller is shown in Fig. 1.

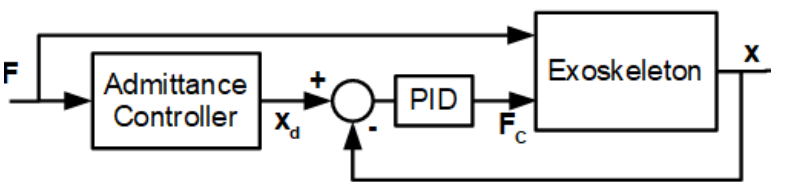

Fig. 1 Basic model of admittance controller

Impedance controllers work oppositely to admittance controllers. In this method, the input is the velocity or position and the system output is a force. This interaction controller is also composed of two loops: an outer velocity or position loop and an inner force loop. For impedance control, an inner feedback loop of torque or force is required while it is a position loop for admittance control. Most electromechanical devices are equipped with position loop control, therefore admittance controllers are simpler to implement than impedance controllers, where a force or torque loop must be designed [2]. Impedance control is more suitable to stiff environments and it is the opposite for an admittance controller.

For the purpose of this research, the environment is a human limb and can be considered compliant. Due to this compliancy, an admittance controller is the most appropriate controller.

In current exoskeletons, interaction control and force/torque or motion control are most often coupled. An exoskeleton controller architecture is firstly composed of an interaction controller. This establishes a relation between the force/motion applied by the user and the error from the robot. The causality depends on if it is an impedance or admittance controller. Then, the force or position controller outputs the desired torque or angle to the robot's joints. Several devices have implemented impedance and force/torque controllers; they are presented in Table I.

Other devices have implemented admittance and position/ velocity controllers. They are presented Table II.
TABLE I

EXOSKELETONS DRIVEN BY IMPEDANCE CONTROLLER

\begin{tabular}{cc}
\hline \hline Device & Developed by \\
\hline ARMin II \& III & $\begin{array}{c}\text { University of Zurich and the } \\
\text { Catholic University of America [5] } \\
\text { Biomedical engineering department } \\
\text { at the Higher Spain Council for } \\
\text { Scientific Research [6] } \\
\text { exoskeleton } \\
\text { University of Twente [7] }\end{array}$ \\
\hline \hline & TABLE II \\
EXOSKELETONS DRIVEN BY ADMITTANCE CONTROLLER \\
\hline Device & Developed by \\
\hline EXO-UL7 & University of California [8] \\
Maryland-Georgetown- & University of Maryland [9] \\
Army Exoskeleton & Northwestern University (USA) [10] \\
\hline
\end{tabular}

In both cases, different improvements could be made. In all aforementioned exoskeletons, the user's physical capacities are not taken into account since all model parameters are fixed. In order to achieve this, some researchers have implemented adaptive or extended methods of control [11]-[13]. According to [2], a conventional impedance/admittance controller is insufficient to interact with the human body. Due to muscle behavior, which experiences nonlinear stiffness modification and is dependent on the motion, using fixed controller parameters cannot achieve the target impedance. This is why for an exoskeleton, the control method requires adaptive (active variable) admittance control or admittance control with forcetracking.

\section{Force-tracking Admittance Controller \\ 3.1. Model}

The system's rise time has a real impact on user comfort. During a fast robot motion, the pressure between the human limb and the device can increase and become painful and restrict blood circulation. The resulting force from this pressure needs to be controlled in order to improve user comfort. Several methods exist for force-tracking in admittance controllers. The first one involves having a virtual time-varying stiffness on the admittance controller. The stiffness is estimated from the difference between the force trajectory and interaction force. This method is comparable to real muscle behavior, since the pressure between a human limb and an object is modifiable due to muscle contraction. An alternative method is to change the desired position trajectory by implementing a desired position loop with a PID controller with force-tracking error as the input.

The method used in this paper is based on using a forcetracking loop with a PID controller in order to directly change the actuator's force trajectory. To simulate and test this controller, a simplified system was considered: two masses $\mathrm{m}_{\text {Robot }}$ and $\mathrm{m}_{\mathrm{H}}$ in translation on a horizontal rail. The first mass represents the robot linked to the frame by a damper $b_{R}$ and a spring $k_{R}$. The second mass represents the human limb. The interaction between the human body and the device is modeled by a spring named $\mathrm{k}_{\text {int. }}$. In Fig. 2 , the $\mathrm{m}_{\text {Robot }}$ mass is the sum of the mass $m_{R}$ and $m_{L s}$. The limb support mass, $m_{L S}$, represents the support used to fix the human body to the robot. This 
component is mounted on the force sensor in order to be able to sense the external force. Fig. 2 displays a model of the human limb linked to the robot by an interaction spring. Damping and stiffness parameters of the human limb are not taken into account in this first model.

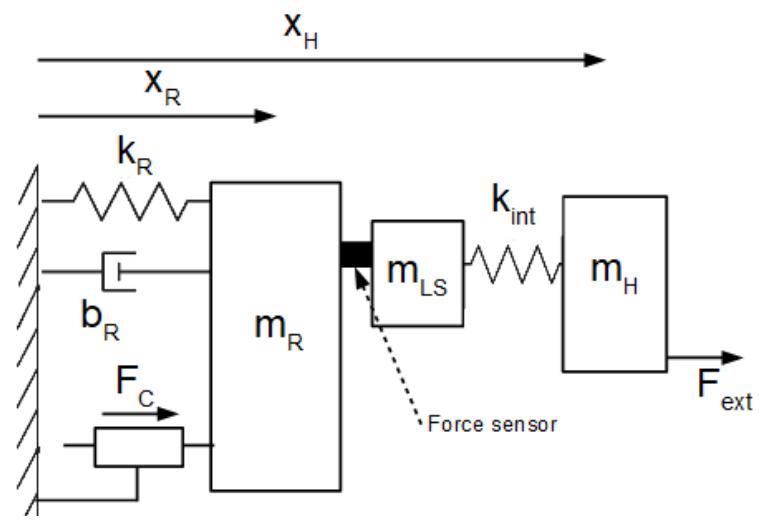

Fig. 2 Device model with limb support and human body inertias

The positions of the device mass and the limb are $x_{R}$ and $x_{H}$, respectively. The actuator and the human body apply forces $F_{C}$ $\& F_{\text {ext }}$. By applying the principle of dynamics on the model shown Fig. 2, (3) gives the motion differential equation of the system.

$$
\left(m_{R}+m_{L S}\right) \ddot{x_{R}}+m_{H} \cdot \ddot{x_{H}}+b_{R} \dot{x_{R}}+k_{R} x_{R}=F_{\text {ext }}+F_{C}
$$

The force $F_{m}$ is the measured force by the load sensor. The expression of this force is given by (4):

$$
F_{m}=F_{\text {ext }}+F_{\text {int }}+m_{H} \cdot \ddot{x_{H}}+m_{L S} \cdot \ddot{x_{R}}
$$

The model of the interaction force $F_{\text {int }}$ is the difference between the robot and the user's limb positions multiplied by the interaction stiffness $k_{\text {int }}$.

$$
F_{\text {int }}=k_{\text {int }} \cdot\left(x_{R}-x_{H}\right)
$$

Therefore, the external force applied by the user is the subtraction between the measured force and forces resulting from the mass motions.

$$
F_{\text {ext }}=F_{m}-\left[k_{\text {int }} \cdot\left(x_{R}-x_{H}\right)+Z_{H}+Z_{L S}\right]
$$

where,

$$
Z_{H}=m_{H} \cdot \ddot{x_{H}} \text { and } Z_{L S}=m_{L S} \cdot \ddot{x_{R}}
$$

Fig. 3 shows the block diagram of the force-tracking admittance controller. The external force passes through the virtual admittance block $Y_{v}$ to set the desired position trajectory. Then, the first PID controller named $C_{l}$ controls a position loop. The inner force loop is controlled by a second PID controller $C_{2}$. Positions $x_{R}$ and $x_{H}$ are measured to estimate the interaction force and compare this value to the force trajectory $F_{d}$.

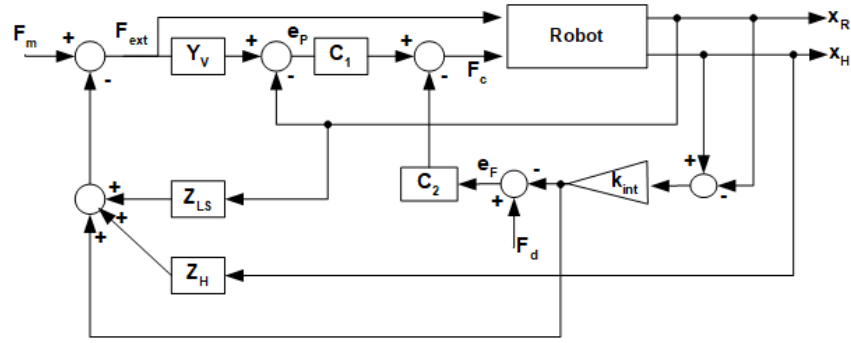

Fig. 3 Block diagram of the force-tracking admittance controller

The model shown in Fig. 2 can be improved by including the damping and stiffness behavior of the human limb. The complete model is displayed in Fig. 4, where $b_{H}$ and $k_{H}$ are the human damping and stiffness coefficients, respectively. The expression of the sensed force is rewritten, including these parameters, in (8). Following the same approach as in the previous equations, the external force is given by (9).

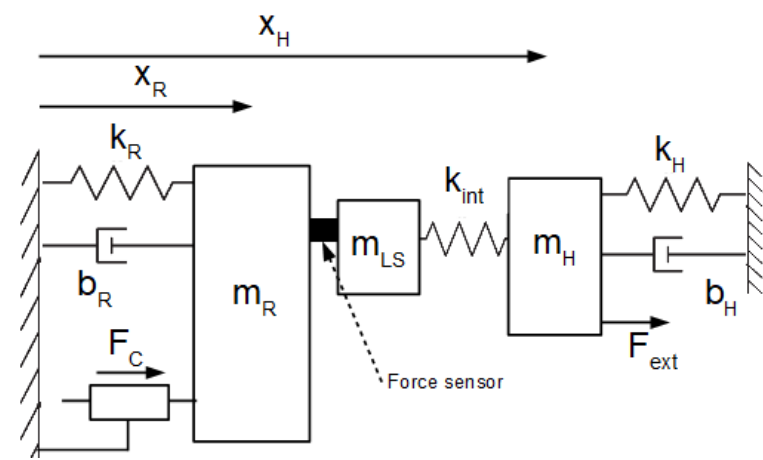

Fig. 4 System model coupled to human limb behavior

$$
\begin{gathered}
F_{m}=F_{\text {ext }}+F_{\text {int }}+m_{H} \cdot \ddot{x_{H}}+b_{H} \cdot \dot{x_{H}}+k_{H} \cdot x_{H}+m_{L S} \cdot \ddot{x}_{R} \\
F_{\text {ext }}=F_{m}-\left[k_{\text {int }} \cdot\left(x_{R}-x_{H}\right)+Z_{H}+Z_{L S}\right]
\end{gathered}
$$

where,

$$
\left\{\begin{array}{c}
Z_{H}=m_{H} \cdot \ddot{x_{H}}+b_{H} \cdot \dot{x_{H}}+k_{H} \cdot x_{H} \\
Z_{L S}=m_{L S} \cdot \ddot{x_{R}}
\end{array}\right.
$$

The system being coupled with the stiffness and the damping of the human limb does not modify the structure of the block diagram shown in Fig. 3. Only the human impedance block changes to the expression in (8).

\subsection{Admittance Controller Performance}

The behavior of the force-tracking admittance controller is determined by the PID gains and virtual dynamics parameters. PID parameters are set up during simulations. The goal in this section is to obtain the admittance parameters $\left(m_{v}, b_{v}, k_{v}, K_{n}\right)$ according to the virtual dynamics requirements $\left(T_{S}(2 \%), M_{P \%}\right.$, $y(\infty))$. To achieve the desired system behavior, the transient response of admittance block $Y_{V}$ has to be short. Indeed, the user must be able to carry out the motion normally and the system must follow and assist in real-time. An underdamped system $(\xi$ $<1)$ is taken for the virtual dynamics in order to have minimized settling time. However, the maximum peak of the desired 
trajectory affects user comfort and makes the system difficult to use. This is why the maximum peak $M_{p}$ is an important parameter for the admittance controller. Equation (11) gives a normalized second-order system.

$$
H=K_{n o r m} /\left(s^{2}+2 \xi \omega_{n} . s+\omega_{n}^{2}\right)
$$

By identification, the damping coefficient $\xi$ and the natural frequency $\omega_{n}$ of the admittance controller are obtained depending on $m_{v}, b_{v}, k_{v}$.

$$
\omega_{n}=\sqrt{\frac{k_{V}}{m_{V}}}, \quad \xi=\frac{b_{V}}{2 \cdot \sqrt{m_{V} \cdot k_{V}}}, \quad K_{\text {norm }}=\frac{K_{n}}{m_{V}}
$$

For an underdamped system, the settling time, the rise time and maximal peak are calculated with:

$$
\begin{gathered}
T_{S(2 \%)}=\frac{4}{\omega_{n} \cdot \xi}, \quad T_{R}=\frac{\pi-\tan ^{-1}\left(\frac{\sqrt{1-\xi^{2}}}{\xi}\right)}{\omega_{n \cdot \sqrt{1-\xi^{2}}}} \\
M_{P}=\left(e^{\frac{-\xi \cdot \pi}{\sqrt{1-\xi^{2}}}}+1\right) \cdot y(\infty)
\end{gathered}
$$

Based on (12) and (13), the settling time can be expressed in terms of $m_{v}$ and $b_{v}$, as seen in (15):

$$
T_{S(2 \%)}=\frac{8 \cdot m_{V}}{b_{V}}
$$

In order to reduce the number of unknown variables, the virtual mass is chosen to be a fixed value that respects the underdamped system conditions. The virtual damping, $b_{v}$, is expressed in terms of settling time and the virtual mass.

$$
b_{V}=\frac{8 \cdot m_{V}}{T_{S(2 \%)}}
$$

Then, to calculate the stiffness $k_{v},(14)$ is used.

$$
\ln \left(\frac{M p}{y(\infty)}-1\right)=\frac{-\pi \cdot \xi}{\sqrt{1-\xi^{2}}}
$$

By substituting (12) into (17), the following equation is obtained:

$$
\frac{\pi^{2}}{\ln ^{2}\left(\frac{M p}{y(\infty)}-1\right)}+1=\frac{4 m_{V} \cdot k_{V}}{b_{V}{ }^{2}}
$$

The settling time can be identified on the right-hand side. Equation (19) is a result of the substitution of (15) into (18):

$$
\left[\frac{\pi^{2}}{\ln ^{2}\left(\frac{M p}{y(\infty)}-1\right)}+1\right] \cdot \frac{8}{T_{S(2 \%)}}=\frac{4 k_{V}}{b_{V}}
$$

Finally, by substituting (16) into (19), the virtual stiffness, $k_{v}$, is estimated:

$$
k_{V}=\frac{16 \cdot m_{V}}{\left.T_{S(2 \%)}\right)^{2}} \cdot\left[\frac{\pi^{2}}{\ln ^{2}\left(M_{P \%}\right)}+1\right]
$$

where,

$$
M_{p}=y(\infty)+y(\infty) \cdot M_{p \%} \rightarrow M_{p \%}=M p / y(\infty)-1
$$

The last admittance parameter to calculate is $K_{n}$, the gain numerator.

$$
K_{n}=k_{V} \cdot y(\infty)
$$

By substituting (20) into (21),

$$
K_{n}=\frac{16 \cdot m_{V}}{T_{S(2 \%)}{ }^{2}} \cdot\left[\frac{\pi^{2}}{\ln ^{2}\left(M_{P \%}\right)}+1\right] \cdot y(\infty)
$$

\section{Simulation and Result Analysis}

The force-tracking admittance controller is tested according to the model displayed Fig. 2. The human leg characteristics (varying stiffness and damping coefficient) are not taken into account in the simulation. The aim of the simulation is to display the effectiveness of adding an inner force-tracking loop in an admittance controller in order to improve user comfort. To show the efficiency of a force-tracking admittance controller, the inclusion of muscle stiffness and damping models is not necessary. For the following simulations the human limb is represented only by a mass $m_{H}$. Instead of the muscle characteristics, a disturbance force is added to the model to analyze the system response. Fig. 5 shows the model diagram used in simulations.

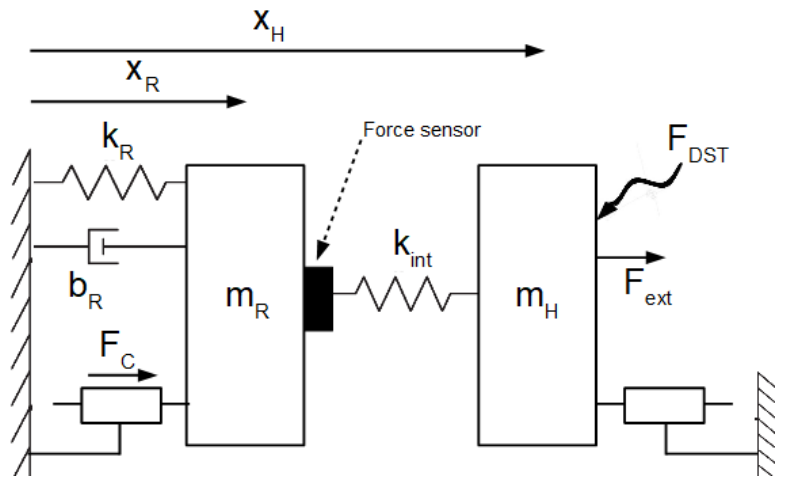

Fig. 5 Simplify model

The fundamental principle of dynamics is applied to each mass in order to obtain the global differential motion equation.

$$
\begin{gathered}
m_{R} \cdot \ddot{x_{R}}+b_{R} \cdot \dot{x_{R}}+k_{R} \cdot x_{R}+k_{\text {int }} \cdot\left(x_{R}-x_{H}\right)=F_{C} \\
m_{H} \cdot \ddot{x_{H}}+k_{\text {int }} \cdot\left(x_{H}-x_{R}\right)=F_{\text {ext }}+F_{D S T}
\end{gathered}
$$

For use in MATLAB, this system is written in state-space form: 


$$
\left\{\begin{array}{c}
\dot{X}=A \cdot\left[\begin{array}{l}
x_{R} \\
x_{H} \\
\dot{x_{R}} \\
\dot{x_{H}}
\end{array}\right]+B \cdot\left[\begin{array}{c}
F_{C} \\
F_{\text {ext }}+F_{D S T}
\end{array}\right] \\
Y=C \cdot\left[\begin{array}{l}
x_{R} \\
x_{H} \\
\dot{x_{R}} \\
\dot{x_{H}}
\end{array}\right]+D \cdot\left[\begin{array}{c}
F_{C} \\
F_{\text {ext }}+F_{D S T}
\end{array}\right]
\end{array}\right.
$$

where,

$$
\begin{gathered}
A=\left[\begin{array}{cccc}
0 & 0 & 1 & 0 \\
\frac{-\left(k_{R}+k_{\text {int }}\right)}{m_{R}} & 0 & 0 & 1 \\
\frac{k_{\text {int }}}{m_{R}} & \frac{b_{R}}{m_{R}} & 0 \\
\frac{k_{\text {int }}}{m_{H}} & \frac{-k_{\text {int }}}{m_{H}} & 0 & 0
\end{array}\right], \quad B=\left[\begin{array}{cc}
0 & 0 \\
0 & 0 \\
\frac{1}{m_{R}} & 0 \\
0 & \frac{1}{m_{H}}
\end{array}\right] \\
C=\left[\begin{array}{llll}
1 & 0 & 0 & 0 \\
0 & 1 & 0 & 0
\end{array}\right], \quad D=\left[\begin{array}{ll}
0 & 0 \\
0 & 0
\end{array}\right]
\end{gathered}
$$

The block diagram shown Fig. 6 is used to drive the simulations. It is assumed that the sensed force $F_{m}$ is equal to the external force. Measured force and disturbance forces are directly applied to the system and pass through the virtual admittance block $Y_{v}$ to set the desired position trajectory.

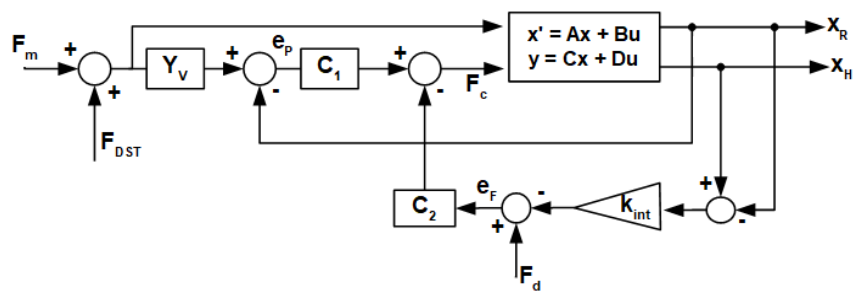

Fig. 6 Simulation controller

\subsection{Simulations}

MATLAB simulations were conducted in order to analyze controller performance. The controller used is the forcetracking admittance controller that was previously simplified and presented (see Fig. 6). Different tests were carried out to highlight the impact of this controller on user comfort. A torque applied by the user and the robot assists the rotation according to an assisting rate coefficient. This motion scenario can be converted to a linear translation with a force as input.

For the first simulation, the force input is a step signal that returns to zero when the motion is finished. This test is composed of a system driven firstly by a simple motion controller and then with an admittance controller. Fig. 7 displays the system time response with these two controllers.

The blue curve represents the time response of the mass $m_{R}$ (see Fig. 7) without the admittance controller. The measured force is simply converted to the desired position with a gain. The pink dash-dotted curve is the $m_{R}$ time response with an admittance controller. The transient response is softer and more damped with the admittance controller. The transient responses are different during the first second and then the signals are similar. This difference is due to the desired position trajectory calculated from the measured force. As shown in Fig. 8, the desired trajectory without the admittance controller is only a multiple of the force input. This trajectory implies an aggressive response with a short rise time.

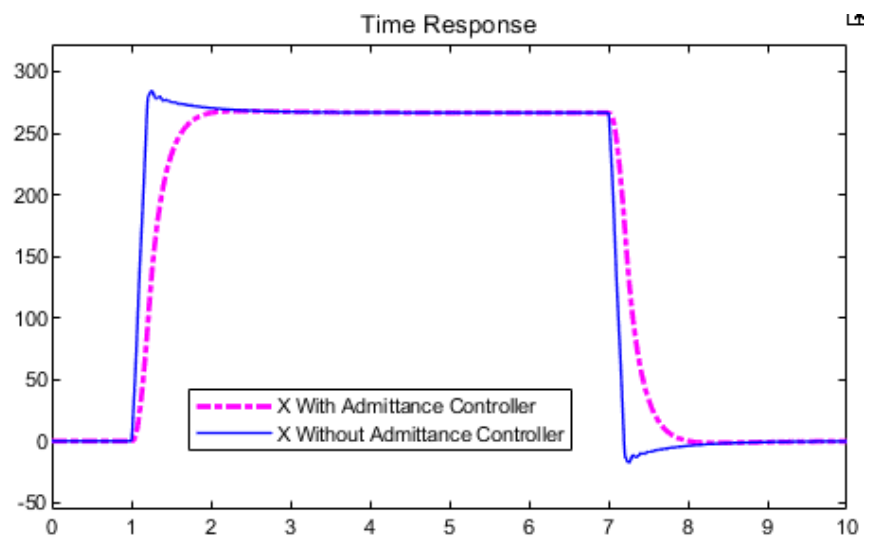

Fig. 7 Time response simulation 1

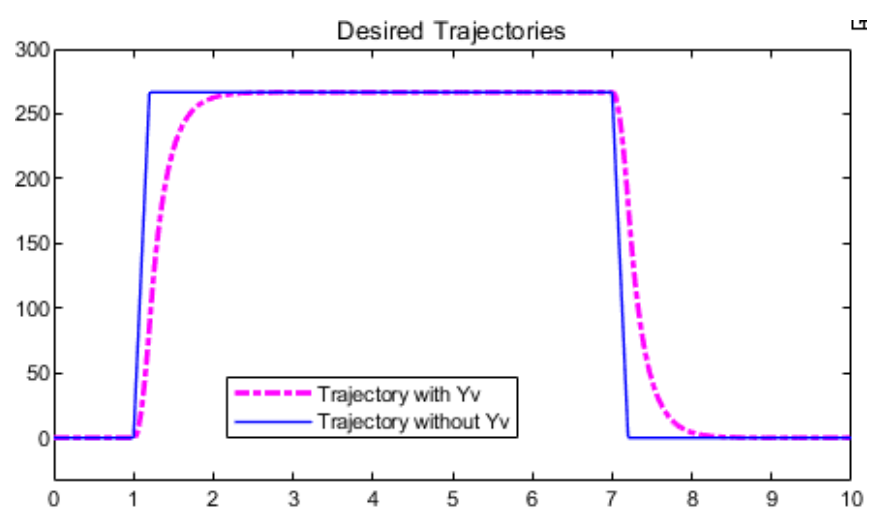

Fig. 8 Desired trajectories simulation 1

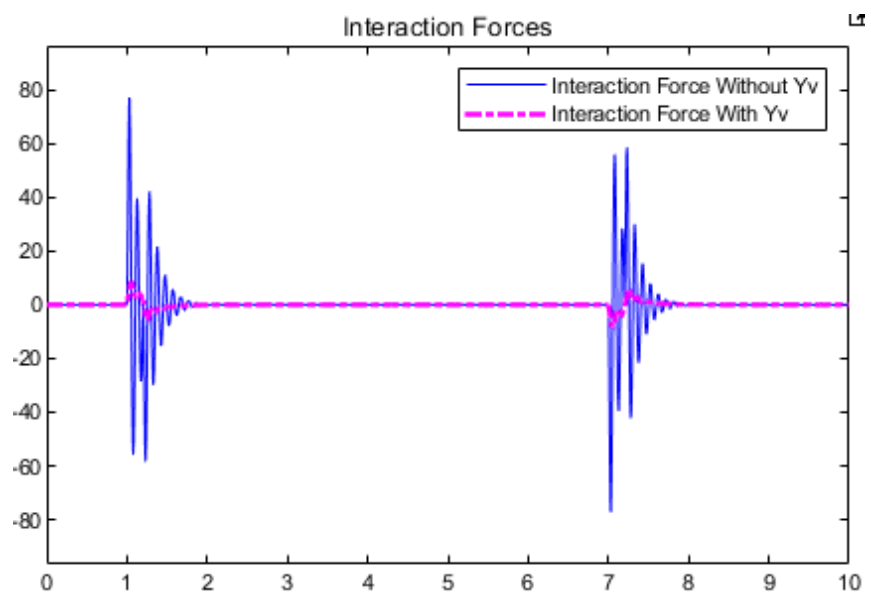

Fig. 9 Interaction forces simulation 1

The high acceleration during the rise time creates a relative pressure between the device and the user. When the desired trajectory is generated through virtual dynamics $\left(Y_{V}\right)$, the acceleration during the transient response is considerably reduced. The desired position trajectory is more damped and the time response is less aggressive than without the admittance controller. The interaction force for both systems is plotted on Fig. 9. The blue curve represents the interaction force of the 
system without the virtual dynamics block $Y_{V}$.

The contact between the human limb and the robot is modeled by a spring. It is for this reason that these interaction forces oscillate. It is interesting to consider the difference between the maximum peak of each signal. Due to the admittance controller, the interaction force is reduced and user comfort is improved.

The next simulation investigates the improvement of the admittance controller. As explained previously, a forcetracking inner loop is added in order to control the interaction force by changing the actuator force trajectory. Fig. 10 shows the time response of the system with and without a forcetracking loop.

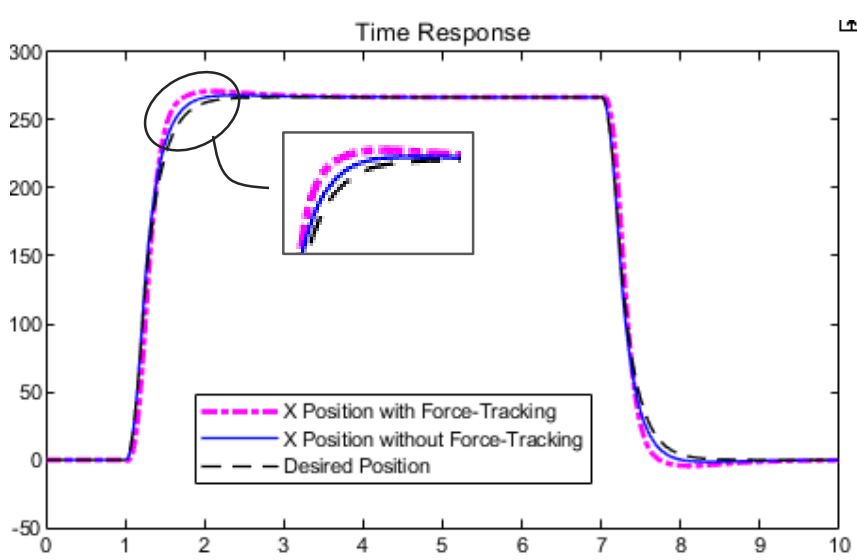

Fig. 10 Time response simulation 2

The black dashed signal is the desired position trajectory, the pink dash-dotted signal is the time response with the forcetracking loop coupled to an admittance controller, and the blue signal is the system response without the force-tracking loop.

In relation to the interaction force, the system's performances with a force-tracking admittance controller are better than with a simple admittance controller. In Fig. 11, the pink dash-dotted signal represents the interaction force between the user and the device with the force-tracking loop.

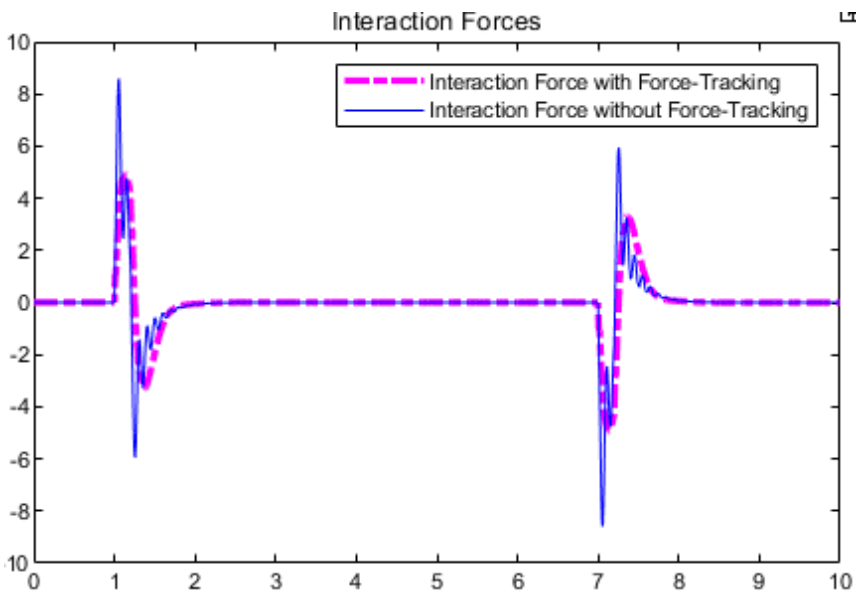

Fig. 11 Interaction forces simulation 2

The interaction force is reduced by $45 \%$ with the force- tracking controller and the oscillations are removed. However, the time response is closer to the desired position for system without the force-tracking loop (see Fig. 10). The position error of each signal is displayed in Fig. 12.

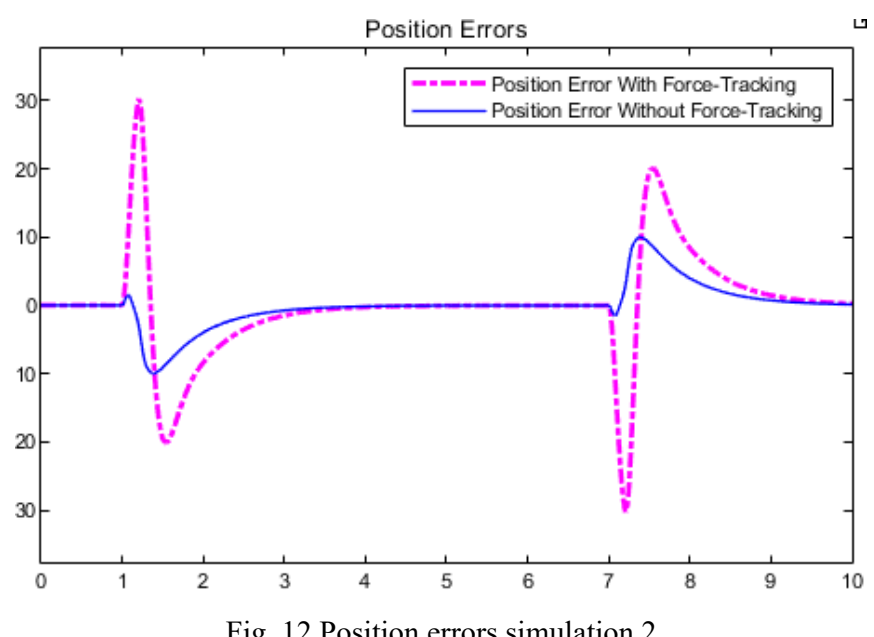

Fig. 12 Position errors simulation 2

The interaction force control increases the position error due to a change in the trajectory of the actuating force. The system acceleration is reduced and the system response is more damped. The inner loop of this controller corrects the interaction force error while the outer loop controls the position error. System performance can be modified by changing the parameters of the PIDs. It is possible to reduce the position error decreasing the efficiency of the PID for the force-tracking loop.

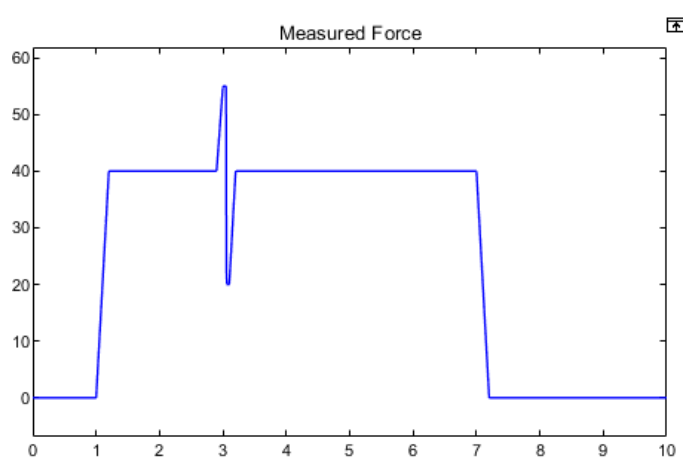

Fig. 13 Measured force scenario simulation 3

When using an exoskeleton or a rehabilitation device, many external factors can affect the system response. One of the most significant factors is human muscle behavior. The user's limb can have a high-varying stiffness capacity, which is useful when the human body needs to react to an unexpected situation. A high force input variation reflects this muscle capacity. Indeed, if the muscle stiffness varies considerably in a short period of time, the force applied to the system will sharply change and modify the desired position trajectory. The objective of the last simulation is to re-create this situation. A disturbance force is added in order to obtain the complete block diagram shown in Fig. 6. Therefore, the measured force scenario is modified and reflects the muscle reaction to an 
unforeseen situation. Fig. 13 depicts the new measured force scenario.

Fig. 14 displays the time response with the disturbance force. The signal legend is the same as in Fig. 10. As observed in simulation 2 , the system response with the force-tracking admittance controller is more deviated from the desired trajectory. This reaction avoids creating a relative pressure between the limb and the robot as much as possible.

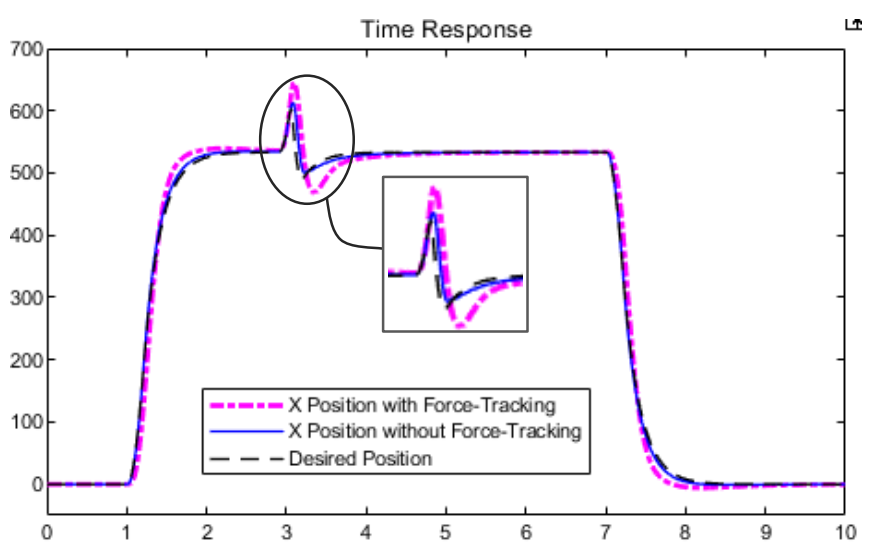

Fig. 14 Time response simulation 3

The force-tracking admittance controller is very effective in this type of situation. During the disturbance, the maximum peak of the interaction force is reduced by $75 \%$ (see Fig. 15).

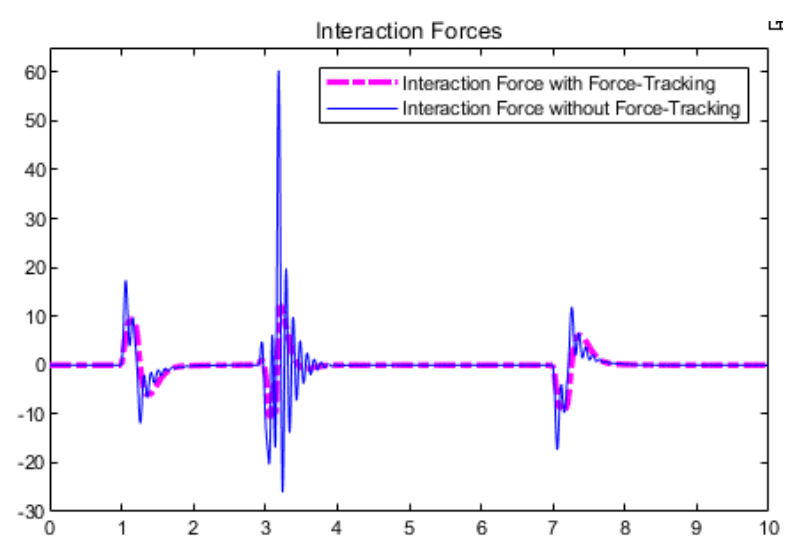

Fig. 15 Interaction forces simulation 3

\subsection{Parameter Analysis}

The system's inputs are the virtual dynamics parameters $\left(m_{V}\right.$, $\left.b_{V}, k_{V}, K_{n}\right)$ and the gains of each PID controller $\left(k_{p}, k_{i}, k_{d}, k_{p f}, k_{i f}\right.$, $k_{d f}$ ). These inputs modify the blocks $Y_{V}, C_{1}$ and $C_{2}$ (see Fig. 6). Each of these parameters affects the system's behavior.

Beginning with the virtual dynamics parameters of the admittance controller, the ratio $K_{n} / k_{V}$ influences the assist rate coefficient. According to the force input, this ratio sets the steady-state value of the desired position trajectory. The higher this ratio, the higher the assist rate. Therefore, the $K_{n} / k_{V}$ value is chosen according to the specific user requirements. The virtual mass $m_{V}$ represents the inertia of the desired position trajectory. If this mass is heavy, the impact of a short and intense force variation is reduced. However, the virtual dynamics block is a second-order system, meaning that a value too high for the $m_{V}$ mass creates oscillations and overshoot in the position trajectory. The last input is the virtual damping coefficient $b_{V}$. This parameter decreases the interaction force during the transient response due to the damping of the position trajectory. As for the virtual mass, it is important to choose a value of $b_{V}$ that avoids creating oscillations that are too strong and overshoot in the desired position. If the $b_{V}$ value is too low, oscillations will appear and the interaction force will be increased. However, the higher the damping, the higher the settling time of the system.

System performance is also determined by the gains of the PID controllers $C_{1}$ and $C_{2}$. The first PID $\left(C_{1}\right)$ controls the position error and the second $\left(C_{2}\right)$ controls the force-tracking error. $C_{l}$ is composed of the gains $k_{p}, k_{i}$, and $k_{d}$, whereas $k_{p f}, k_{i f}$, and $k_{d f}$ are the gains for controller $C_{2}$. It is possible to adjust the system behavior due to the inclusion of these PIDs. The control strategy can be focused on interaction force control, on position control, or it can be a compromise of both. Having optimal control of both interaction force and position is impossible due to the causality of force/acceleration. By decreasing the gain $k_{p f}$, the efficiency of the controller $C_{2}$ is reduced, thereby decreasing the position error and increasing the force-tracking error. $k_{i f}$ affects system behavior in the same way as $k_{p f}$. The gain $k_{d f}$ creates oscillations and makes the system unstable if it is nonzero. The integral gain $k_{i}$ of the motion controller does not affect the force-tracking error. If this gain is too low, the steadystate error rises and if it is too high, overshoot rises. The proportional gain $k_{p}$ acts symmetrically to $k_{p f}$ on the system behavior. By increasing $k_{p}$, the efficiency of controller $C_{1}$ is improved, the position error decreases and the force-tracking error rises. The derivative gain $k_{d}$ is necessary to avoid creating oscillations of the system time response. Moreover, this gain has the same impact as $k_{p}$ on the behavior of the system.

\section{Conclusion}

Exoskeleton technology has significantly progressed in recent years. Though, the problem of user comfort remains to be solved, improving robot control methods is one of the possible solutions to overcome this challenge. In this paper, a controller based on a force-tracking admittance method has been developed and tested to show its effectiveness in reducing the relative pressure between the user and the robot during transient regimes or in face of disturbances. However, the tests were only performed as simulations on a 3D model of a test bench. Future experimental tests must be carried out on a physical test bench to compare the results with those obtained during simulations.

\section{References}

[1] A. Seguin, "Experimental Characterisation of Polyurethane Foam-Based Magnetorheological Elastomers", Thesis submission, Ottawa-Carleton Institute for Biomedical Engineering, University of Ottawa, Canada, 2018.

[2] H. F.N. Al-Shuka, S. Leonhardt, W. Zhu, R. Song, C. Ding and Y. Li, "Active Impedance Control of Bioinspired Motion Robotic Manipulators: An Overview", in Bionics and Biomechanics, Hindawi, Vol 2018, Article 
ID 8203054,18 October 2018

[3] K. Anam, A. Al-Jumaily, “Active Exoskeleton Control Systems: State of the Art", International Symposium on Robotics and Intelligent Sensors 2012 (IRIS 2012), Procedia Engineering vol 41 pp.988-994.

[4] N. Berezny, "Design and Implementation of a Novel Rehabilitation Robot for Acute Stroke Patients", thesis submitted to the University of Ottawa, Canada, Dept. Mechanical \& Aerospace Engineering, September 20219.

[5] T. Nef, M. Mihelj, G. Kiefer, C. Oerndl, R. Müller, R. Riener. "ARMin Exoskeleton for Arm Therapy in Stroke Patients", International Conference on Rehabilitation Robotics, IEEE, June 2007.

[6] E. Rocon, J.M. Belda-Lois, A.F. Ruiz, M. Manto, J.C. Moreno, and J.L. Pons, "Design and Validation of a Rehabilitation Robotic Exoskeleton for Tremor Assessment and Suppression", Transactions on neural systems and rehabilitation engineering conference, vol. 15, no. 3, September 2007.

[7] J. Veneman, R. Kruidhof, E. G. Hekman et al, "Design and Evaluation of the LOPES Exoskeleton Robot for Interactive Gait Rehabilitation", presented at transactions on neural systems and rehabilitation engineering conference, vol. 15, no. 3, September 2007.

[8] W. Yu, J. Rosen, X. Li, "PID Admittance Control for an Upper Limp Exoskeleton". In American Control Conference, July 2011.

[9] C. Carignan, J. Tang, and S. Roderick, "Development of an Exoskeleton Haptic Interface for Virtual Task Training”, in International Conference on Intelligent Robots and Systems, IEE, October 2009.

[10] G. Aguirre-Ollinger, J. Edward Colgate, Michael A. Peshkin and Ambarish Goswami, "Design of an active one-degree-of-freedom lowerlimb exoskeleton with inertia compensation", published in The Internation Journal of Robotics Research, December 2010.

[11] G. Li, H. Huang, and B. Li, "Robust Adaptive Force Tracking Impedance Control for Robotic Capturing of Unknown Objects", in International Conference on Intelligent Robot and Applications, Vol 12, August 2019.

[12] K. Lee and M.Buss, "Force Tracking Impedance Control with Variable Target Stiffness", presented at World Congress The International Federation of Automatic Control, Vol 17, pp.6751-6756, July 2008.

[13] K. Kiguchi and Y. Hayashi, "An EMG-Based Control for an Upper-Limb Power-Assist Exoskeleton Robot", in Transactions on Systems, MAN, and Cybernetics-Part B, Vol 42, pp.1064-1071, August 2012.

\section{Creative Commons Attribution License 4.0 (Attribution 4.0 International, CC BY 4.0)}

This article is published under the terms of the Creative Commons Attribution License 4.0

https://creativecommons.org/licenses/by/4.0/deed.en_US 